\title{
Celiac disease with acute cerebellar stroke as first presentation: A case report
}

\author{
1,2 Maria Maddalena Sirufo, ${ }^{1,2}$ Enrica Maria Bassino, ${ }^{1,2}$ Francesca De Pietro, ${ }^{1}$ Lina \\ Maria Magnanimi, ${ }^{1,2}$ Lia Ginaldi, ${ }^{1,2}$ Massimo De Martinis \\ ${ }^{1}$ Department of Life, Health and Environmental Sciences, University of L'Aquila, Italy; ${ }^{2}$ Allergy and \\ Clinical Immunology Unit, Center for the diagnosis and treatment of Osteoporosis, AUSL 04 Teramo, \\ Italy
}

\begin{abstract}
Celiac disease is an immune disorder occurring in response to ingestion of gluten in genetically predisposed individuals. It is a complex multiorgan disease with possible neurological involvement. Thrombotic events can occur but rarely as presenting symptom. We describe the case of a young man admitted to the Stroke Unit for worsening headache, nausea, vomiting, unsteadiness, dysarthria, and dysmetria. Brain CT scan showed an ischemic hypodense lesion of the right cerebellar hemisphere with compression of the adjacent IV ventricle. Studies to determine the etiology of stroke showed positive serology for celiac disease. On follow up he has completely recovered clinically. He is on gluten-free diet and the serology is now negative. Our case demonstrated that in young stroke, even without gastrointestinal symptoms, celiac disease should be considered a possible differential diagnosis and the appropriate serology test performed.
\end{abstract}

Keywords: Stroke, celiac disease, thrombosis, neurological manifestation, gluten

\section{INTRODUCTION}

Celiac disease $(\mathrm{CD})$ is an enteric autoimmune disease in genetically susceptible individuals triggered by the ingestion of gluten-containing grains. The main target of the immunological insult is the gastrointestinal tract but $\mathrm{CD}$ is systemic disease with possible involvement in any organ or tissue of the body. About $0.7 \%$ of the population worldwide suffers from the disease. ${ }^{1}$

The varied presentation and vague symptoms can make the diagnosis of CD difficult, and delay in diagnosis is common. In more than $50 \%$ of the cases in adults, the patients have significant extra-intestinal involvement even in the absence of the typical CD gastrointestinal symptoms. ${ }^{2}$

$\mathrm{CD}$ can manifest with several neurologic symptoms, these include cerebellar ataxia, myoclonic ataxia, ischemic stroke, epilepsy, chronic neuropathies, progressive leukoencephalopathy, dementia, depression, and anxiety. ${ }^{3-7}$

Stroke was the third most common cause of disability and the second most common cause of death worldwide in $2013 .{ }^{8}$ There is an increased risk of stroke in $\mathrm{CD}$ patients ${ }^{9}$ and stroke can also be the presenting symptom of an otherwise silent CD. ${ }^{10-12} \mathrm{CD}$ should be regarded as a possible etiology of stroke particularly among the young, even among those who are without gastrointestinal symptoms, although among the neurologic manifestations of $\mathrm{CD}$, stroke is rare and there are not many reports in the literature.

\section{CASE REPORT}

A 36 years old Moroccan man was taken to the Emergency Department in Teramo, Italy and admitted to the Stroke Unit for worsening headache, nausea, vomiting, unsteadiness, dysarthria and ataxia. He was alert but was uncooperative. The neurologic evaluation showed the presence of bilateral nystagmus more evident on gazing to the right side, ataxic finger-nose test both sides, worse on the right, and a positive Rhomberg test. On the second day, a convergent left squint, left central facial palsy was observed followed by impaired consciousness. Further clinical history revealed a previous hospitalization for an episode of diarrhea upon return from Morocco. A brain CT scan on the second day 
showed an ischemic hypodense lesion of the right cerebellar hemisphere with compression of the adjacent IV ventricle. (Figure 1B)

A detailed family history did not reveal any further relevant information; in particular there was no past history of cardiovascular diseases, diabetes or dyslipidemia. He did not smoke tobacco.
Laboratory analyses including complete blood count, lipids, C reactive protein, erythrocyte sedimentation rate, renal, liver and thyroid function, glucose, hemoglobin A1c, cobalamin, vitamin B12, HIV, hepatitis B and C, syphilis, sickle screen, thrombophilia screening, autoimmunity and toxicology screening were all in the normal
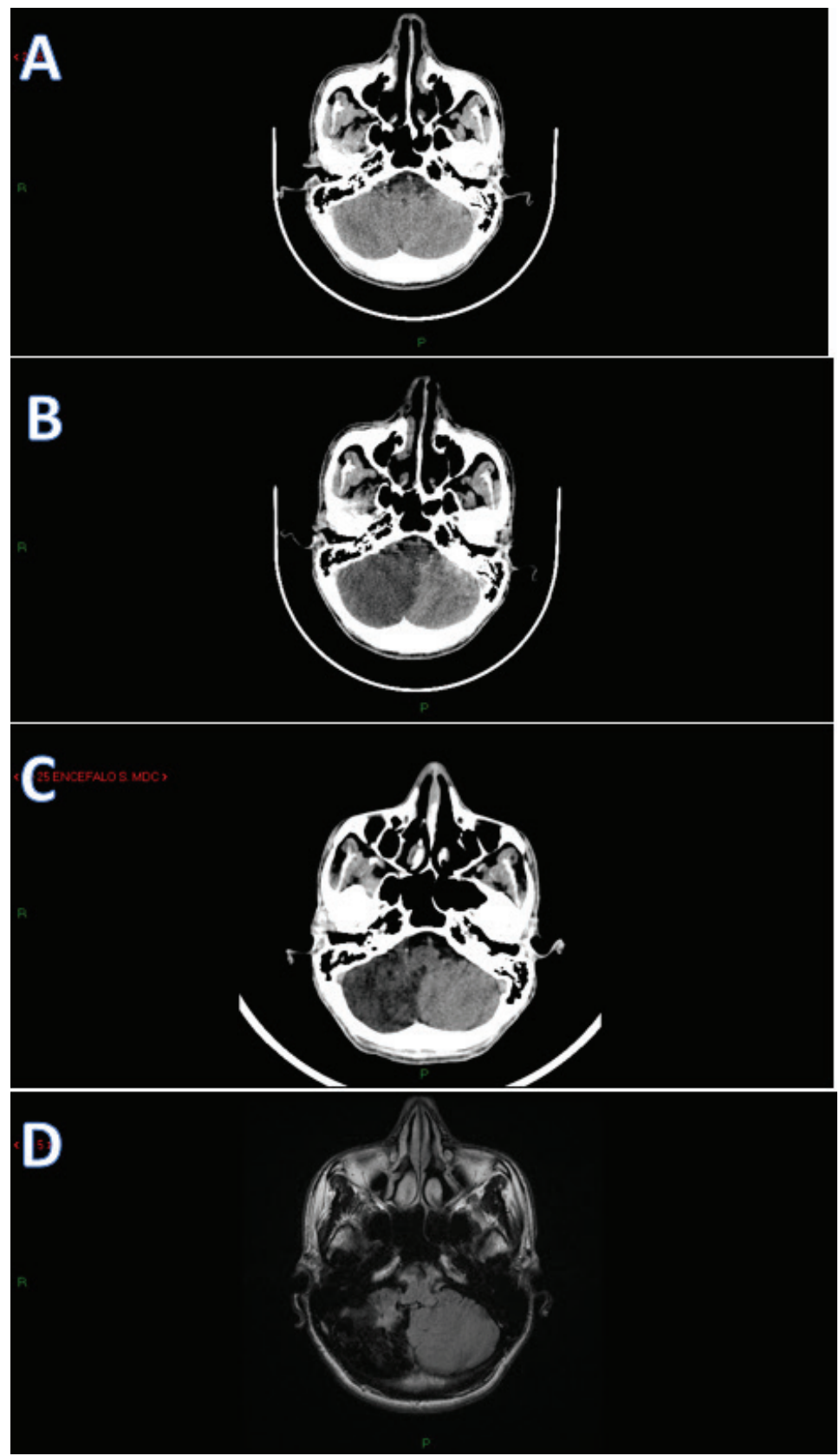

Figure 1. Brain CT scan: A. at the Emergency Department on the first day was normal. B. On the second day the scan showed a large ischemic hypodense area of the right cerebellar hemisphere C. After one month, the right cerebellar infarct lesion has reduced in size. D. Brain MRI after three years showing that the previous infarct has become encephalomalacia. 
ranges. Anti-transglutaminase antibodies ( $\operatorname{IgA}$ $123 \mathrm{UI} / \mathrm{ml}$ ), anti-endomysium antibodies Ig A positivity and anti-gliadin antibodies (IgA $61 \mathrm{UI} /$ $\mathrm{ml})(\mathrm{IgG} 58 \mathrm{UI} / \mathrm{ml})$ were strongly positive. There was presence of alleles HLA-DQ2 (homozygosity) and HLA-DQ8. Cardiac investigations did not reveal any potential cardioembolic sources, with normal electrocardiogram, telemetry monitoring and transthoracic echocardiography with bubble study. Carotid artery duplex scan, and computed tomography angiography were also normal. MRI performed later confirmed the right cerebellar ischemic lesion, but no corresponding arterial lesion in the MR angiography (Figure 2).

On follow up, he has completely recovered clinically with no recurrence of similar episode. He was on gluten-free diet and anti-transglutaminase, anti-endomysium and anti-gliadin antibodies later became negative.

\section{DISCUSSION}

The etiology of ischemic stroke in young adults and adolescents is diverse and varies according to age and geographic region. Modifiable risk factors such as hypertension, dyslipidemia, and cigarette smoking have been reported to be common in young stroke patient. ${ }^{13}$ For optimal diagnosis and management of stroke in young adults a "heart to head" approch is recommended, in addition to the standard vascular risk factors. Alternative etiologies need to be considered, including investigating the rare causes. ${ }^{14,15}$ An increased risk for ischemic stroke has been reported in patients with celiac disease. ${ }^{16}$ Increased risk for stroke can persist even when taking gluten-free diet; transient ischemic attack and ischemic infarct may occur in asymptomatic CD untreated subjects. ${ }^{9}$ On the other hand, there are few reports of ischemic stroke associated with CD in literature in asymptomatic subjects. ${ }^{10-12}$ This increased risk may be related to the underlying autoimmune pathology that increase the hypercoagulable and thromboembolic risk. ${ }^{17}$

Our patient was young, he was not a smoker with no other cardiovascular risk factors. The relationship between the single episode of diarrhea that led to admission and CD is uncertain. This case described highlights the importance of considering $\mathrm{CD}$ in the differential diagnosis of unexplained ischemic stroke, especially in young adults with no thrombophilia or known cardiovascular risks even in the absence of gastrointestinal symptoms. The prevalence of $\mathrm{CD}$ and the reports of cases presenting with extraintestinal symptoms is rising.
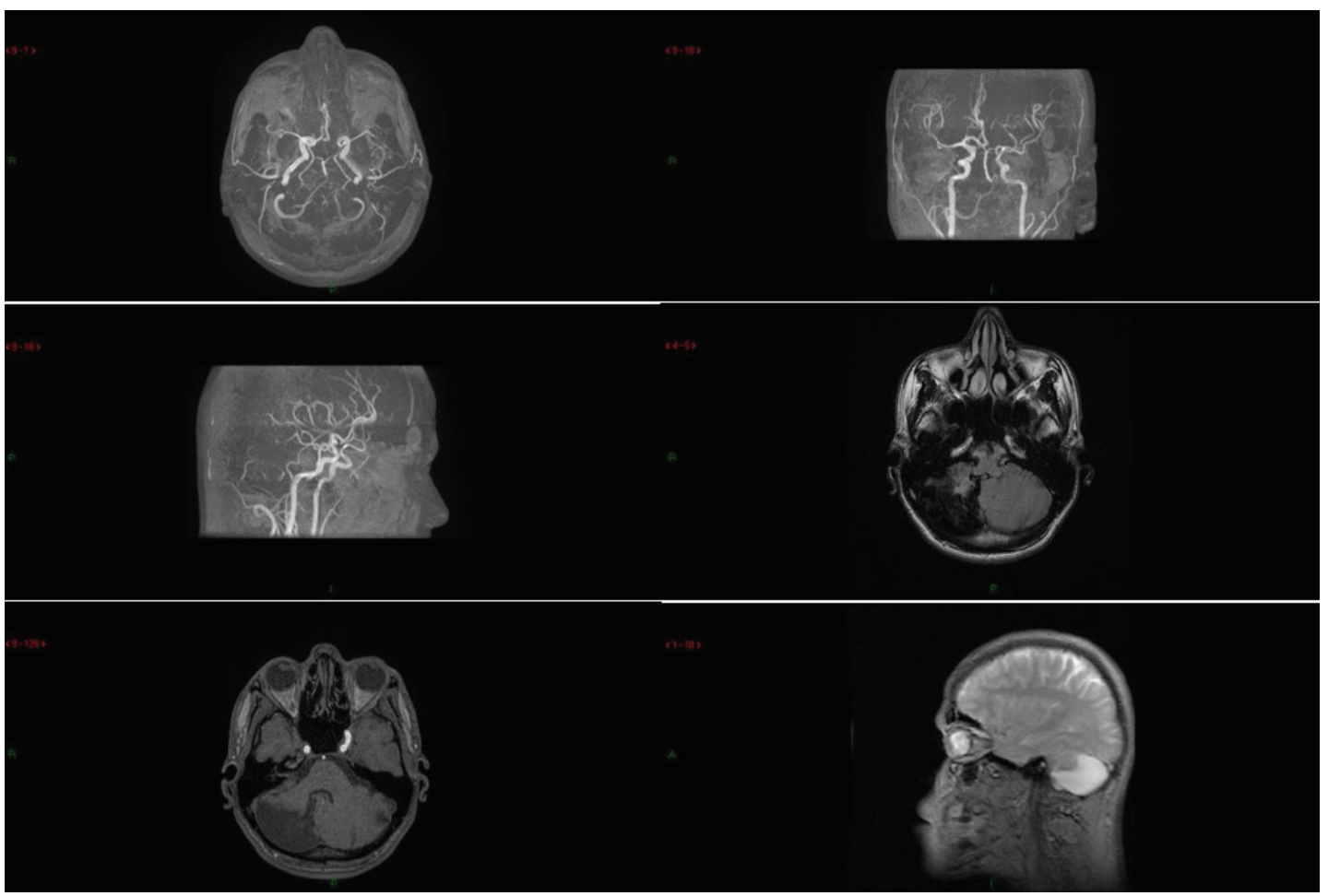

Figure 2. The MRA of the cerebral arteries do not show any abnormality relevant to the right cerebellar infarct. The MRI brain on follow up shows that the right cerebellar infarct has become encephalomalacia. 
Diarrhea, abdominal bloating, weight loss, and nutrient malabsorption are the gastrointestinal manifestations of $\mathrm{CD}$, while abnormalities in liver function tests, iron-deficiency anemia, fatigue, skin disorders, bone disease and a wide spectrum of psychiatric and neurological disorders are some of the extraintestinal manifestations. Neurologic manifestations occur in about $10-20 \%$ of celiac patients and may either precede or follow $\mathrm{CD}$ diagnosis. The pathophysiology of neurologic manifestations associated with $\mathrm{CD}$ remains largely elusive, although nutritional deficiencies and cross-reacting immune responses are postulations. ${ }^{18}$ There was no particular symptom in our patient to indicate a diagnosis of $\mathrm{CD}$, as the history of diarrhoea was realated to his return from Morocco. The diagnosis of CD was attained in the context of investigation of young stroke. It is therefore necessary to be exhaustive in the search for causes of young stroke. ${ }^{19}$

To establish gluten sensitivity as a possible cause of neurological illness the antigliadin antibody test is a low cost and easily available test, that should be included in the investigation of any patient with neurological dysfunction of unknown cause..$^{20,21}$

Our report confirm that particularly in the young, even in the context of the absence of gastrointestinal symptoms, when there is no obvious etiology, $\mathrm{CD}$ should be considered as a possible cause. This is particularly important as $\mathrm{CD}$ is a potentially treatable.

\section{DISCLOSURE}

Ethical statement: The study was conducted in compliance with the established rules of our Internal Review Board and conducted in accordance with the 1975 Helsinki Declaration and its subsequent amendments. The patient provided his written informed consent to participate and for the publication of this report and any associated image.

Financial support: None

Conflicts of interest: None

\section{REFERENCES}

1. Glissen Brown JR, Singh P. Coeliac disease. Pediatr Int Child Health 2018; 13:1-9

2. Pennisi M, Bramanti A, Cantone M, Pennisi G, Bella R, Lanza G. Neurophysiology of the "celiac brain": disentangling gut-brain connections. Front. Neurosci 2017; 11:1-13.
3. Rani U, Imdad A, Beg M. Rare neurological manifestation of celiac disease. Case Rep Gastroentero 2015; 9: 200-5

4. Hadjivassiliou M, Rao DG, Grìnewald RA, et al. Neurological dysfunction in coeliac disease and non-coeliac gluten sensitivity. Am J Gastroenterol 2016; 111(4): 561-7.

5. Pacitto A, Paglino A, Di Genova L, et al. Celiac disease presenting with peripheral neuropathy in Children: A case report. Int J Environ Res Public Health 2017 ; 14(7):785.

6. Gao Z, Varma DD, Patel S, Lee A, Chen C. Visual loss secondary to bioccipital calcifications associated with coeliac disease. Neuroophthalmology 2015; 39(6):277-80

7. Rouvroye MD,Zis P, Van Dam AM, Rozemuller AJM, Bouma G, Hadjivassiliou M. The neuropathology of gluten-related neurological disorders: A systematic review. Nutrients 2020;12(3):822.

8. Valery L. Feigin, Bo Norrving, George A. Mensah. Global burden of stroke. Circ Res 2017; 120: 439-48.

9. Ludvigsson JF, West J, Card T, Appelros P. Risk of stroke in 28000 patients with celiac disease: a nationwide cohort study in Sweden. J Stroke Cerebrovasc Dis 2012; 21: 860-7.

10. Poulin W, Gaerner S, Cordeanu EM, Mirea C, Andres E, Stephan D. Stroke revealing celiac disease associated with multiple arterial thrombotic locations. La Presse Medicale 2015; 44(5):537-8.

11. Beyrouti R, Mansour M, Kacem A, Derbali H. Recurrent cerebral venous thrombosis revealing celiac disease: an exceptional case report. Acta Neurol Belg 2017; 117:341-3.

12. Rachid B, Zouhayr S, Naima C, Ouafae M, Faouzi B. Accident vasculaire cerebral ischemique revelant une maladie coeliaque. Pan African Med J 2010; 5:2.

13. Ciaccio EJ, Lewis SK, Biviano AB, Iyer V, Garan $\mathrm{H}$, Green PH. Cardiovascular involvement in celiac disease. World J Cardiol 2017; 9 (8): 652-66.

14. Smajlović D. Strokes in young adults: epidemiology and prevention. Vasc Health Risk Manag 2015;11:15764.

15. Stack CA, Cole JW. Ischemic stroke in young adults. Curr Opin Cardiol 2018 ;33(6):594-604.

16. Polivka J Jr, Polivka J, Pesta M, et al. Risks associated with the stroke predisposition at young age: facts and hypotheses in light of individualized predictive and preventive approach. EPMA J 2019 ;10(1):81-99.

17. Lerner A, Blank M. Hypercoagulability in celiac disease. An update. Autoimmunity Rev 2014; 13:113841.

18. Falato E, Capone F, Ranieri F, et al. Celiac disease diagnosed in an older adult patient with a complex neuropsychiatric involvement: A case report and review of the literature. Brain Sci 2020;10(7):426.

19. Kempster PA. Looking for clues. J Clin Neurosci 2006;13(2):178-80.

20. Hadjivassiliou M, Gibson A, Davies-Jones GA, Lobo AJ, Stephenson TJ, Milford-Ward A. Does cryptic gluten sensitivity play a part in neurological illness? Lancet 1996;347(8998):369-71.

21. Unsworth DJ. Gluten sensitivity and neurological dysfunction. Lancet 1996;347(9005):903-4. 